Development of Sensors and Sensing Technology for Hydrogen Fuel Cell Vehicle Applications

E. L. Brosha, P. K. Sekhar, R. Mukundan, T. Williamson, F. H. Garzon, L. Y. Woo, R. R. Glass

January 7,2010

ECS Transactions: Fuel Cell Seminar and Exposition 2009 
This document was prepared as an account of work sponsored by an agency of the United States government. Neither the United States government nor Lawrence Livermore National Security, LLC, nor any of their employees makes any warranty, expressed or implied, or assumes any legal liability or responsibility for the accuracy, completeness, or usefulness of any information, apparatus, product, or process disclosed, or represents that its use would not infringe privately owned rights. Reference herein to any specific commercial product, process, or service by trade name, trademark, manufacturer, or otherwise does not necessarily constitute or imply its endorsement, recommendation, or favoring by the United States government or Lawrence Livermore National Security, LLC. The views and opinions of authors expressed herein do not necessarily state or reflect those of the United States government or Lawrence Livermore National Security, LLC, and shall not be used for advertising or product endorsement purposes. 


\section{Development of Sensors and Sensing Technology for Hydrogen Fuel Cell Vehicle Applications}

Eric L. Brosha, Praveen K. Sekhar, Rangachary Mukundan, Todd Williamson, and

Fernando H. Garzon

Los Alamos National Laboratory

MPA-11, Sensors and Electrochemical Devices Group

P.O. Box 1663, MS D429

Los Alamos, New Mexico 87545

Leta Y. Woo and Robert R. Glass

Lawrence Livermore National Laboratory

P.O. Box 808, Mail code: L-367

Livermore, California 94551

One related area of hydrogen fuel cell vehicle (FCV) development that cannot be overlooked is the anticipated requirement for new sensors for both the monitoring and control of the fuel cell's systems and for those devices that will be required for safety. Present day automobiles have dozens of sensors on-board including those for IC engine management/control, sensors for state-of-health monitoring/control of emissions systems, sensors for control of active safety systems, sensors for triggering passive safety systems, and sensors for more mundane tasks such as fluids level monitoring to name the more obvious. The number of sensors continues to grow every few years as a result of safety mandates but also in response to consumer demands for new conveniences and safety features.

Some of these devices (e.g. yaw sensors for dynamic stability control systems or tire pressure warning RF-based devices) may be used on fuel cell vehicles without any modification. However the use of hydrogen as a fuel will dictate the development of completely new technologies for such requirements as the detection of hydrogen leaks, sensors and systems to continuously monitor hydrogen fuel purity and protect the fuel cell stack from poisoning, and for the important, yet often taken for granted, tasks such as determining the state of charge of the hydrogen fuel storage and delivery system. Two such sensors that rely on different transduction mechanisms will be highlighted in this presentation. The first is an electrochemical device for monitoring hydrogen levels in air. The other technology covered in this work, is an acousticbased approach to determine the state of charge of a hydride storage system.

\section{Electrochemical $\mathrm{H}_{2}$ Gas Sensors for Fuel Cell Safety}

The DOE Hydrogen Codes and Standards program is part of an international effort to develop and establish a global technical regulation for Hydrogen/Fuel Cell Vehicles (HFCV) that attains equivalent levels of safety as those for conventional gasoline powered vehicles but does not restrict future technologies. Based on a comparison of existing regulations and standards of HFCV with conventional vehicles, the goal of the program is to investigate and consider the main differences in safety and environmental aspects and what items need to be regulated based 
on justification. Parallel work is being conducted by other Federal Agencies such as the Department of Transportation (DOT) and National Highway Traffic Safety Administration (NHTSA) with the initiation of a four-year research program on hydrogen fuel cell vehicles that includes studies on electrical isolation, failure mode effects analysis (FMEA) for compressed gas HFCVs, evaluation and comparative analysis of existing and draft regulations and standards, and compressed hydrogen fuel container integrity testing.

One important requirement for implementation of HFCVs is an inexpensive, reliable, and long-lived sensor (usual requirements for present day vehicles) for the detection of $\mathrm{H}_{2}$. There are several sensor technologies that have been developed to detect hydrogen, including deployed systems to detect leaks in manned space systems and hydrogen safety sensors for laboratory and industrial usage. The most common technologies are based on catalytic bead combustible gas sensors, infrared absorption, electrochemical oxidation of hydrogen, or the resistance/capacitance change of Pd-based materials. While the Pd-based sensors have excellent selectivity, they need frequent calibration like other similar resistance measurement-based technologies. On the other hand, electrochemical sensors that operate close to room temperature (similar to commerciallyavailable $\mathrm{CO}$ sensors) exhibit better stability, but possess short life times and are susceptible to poisoning or humidity cross interference. In contrast, electrochemical devices that utilize high temperature-based ceramic electrolytes are largely unaffected by changes in humidity and are more resilient to electrode or electrolyte poisoning.

As part of the Hydrogen Codes and Standards program, LANL and Lawrence Livermore National Laboratory (LLNL) are working together to develop and test inexpensive, zirconiabased, mixed potential sensors for $\mathrm{H}_{2}$ detection in air. This type of device is a derivative of the zirconia $\mathrm{O}_{2}$ sensor found in every gasoline-powered vehicle sold today. Previous work conducted at LLNL showed that indium tin oxide (ITO) electrodes produced a stable mixed potential response in the presence of up to $5 \%$ of $\mathrm{H}_{2}$ in air with no response to $\mathrm{CO}_{2}$ or water vapor $(1,2)$. The sensor also showed desirable characteristics with respect to response time and resistance to aging, and degradation due to thermal cycling. In past year, we have worked to prepare new ITO-based electrochemical devices using sensor configurations developed at LANL for the preparation of stable electrochemical interfaces for mixed potential type sensor applications (37). Most recent development work outlined in this extended abstract has focused on the application of commercial ceramic sensor manufacturing methods to prepare devices with substantially less power requirements and stable, long term performance.

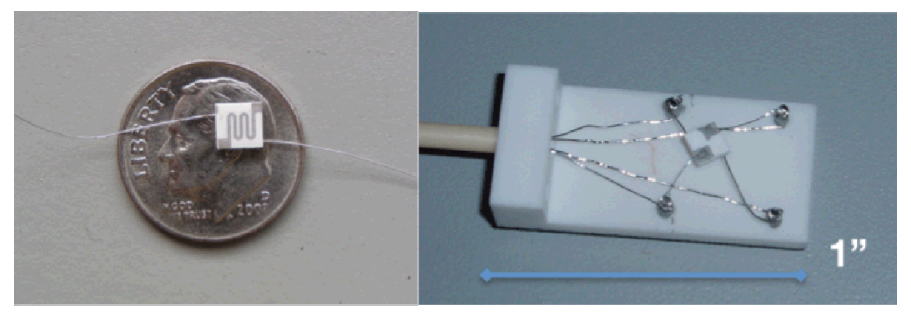

Figure 1. Miniature (4mm x 4mm) ITO/YSZ/Pt $\mathrm{H}_{2}$ mixed potential sensor prepared for LANL by ESL ElectroScience using commercial ceramics tape casting and screen-printing methods. Integrated Pt resistive heater shown on left. 
The miniaturized, and low power hydrogen sensor prototype developed at LANL was based on the LLNL 'Indium Tin Oxide (ITO) / Yttria-Stabilized Zirconia (YSZ) / Platinum (Pt)' configuration. The device fabricated on an alumina substrate integrates a resistive Pt heater to achieve precise operating temperature and minimize heterogeneous catalysis.

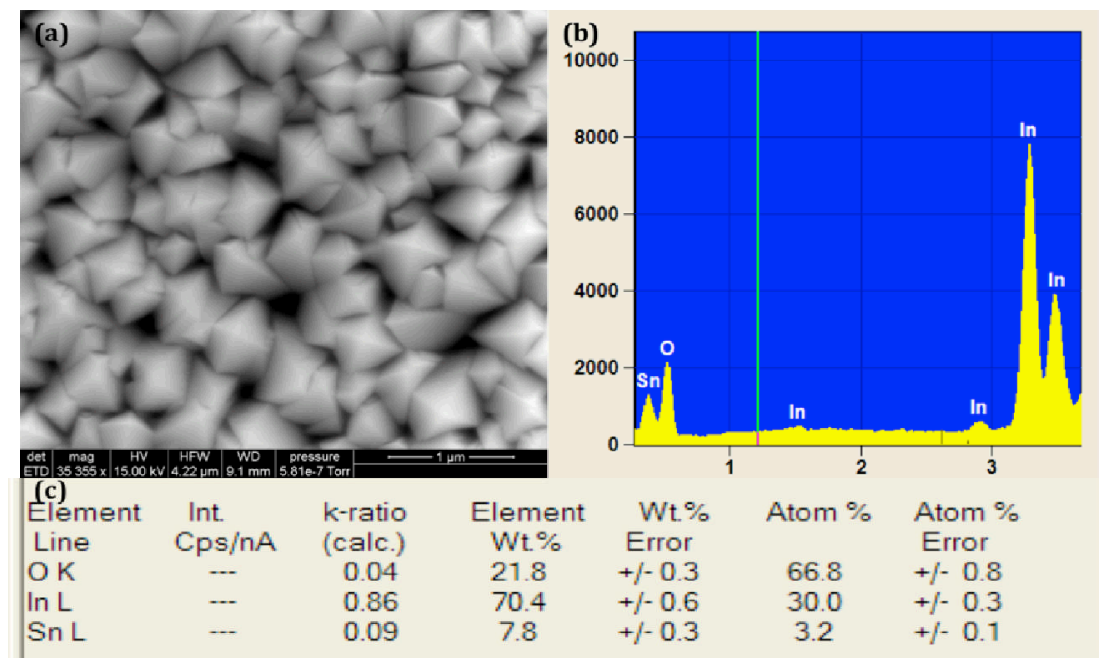

Figure 2. Morphology and composition of the ITO electrode: (a) SEM showing the pyramidal microstructure of the ITO surface, (b) energy dispersive spectrum (EDS) indicating the presence of In, Sn, and O, and (c) EDS-Quant Analysis showing the atomic stoichiometry of the sputtered ITO film.

The ITO electrode was sputter deposited onto the alumina substrate, while YSZ electrolyte was deposited using electron beam technique. Upon inspecting the as-sputtered ITO electrode surface, pyramidal microstructures were observed (Figure 2a). The EDS image (Figure 2b) confirms the presence of $\mathrm{In}, \mathrm{Sn}$ and $\mathrm{O}$ with an atomic stoichiometry of 30\%, 66.8\% and 3.2\% respectively (Figure 1c). Also, the ITO film was annealed at 450, 600 and $700^{\circ} \mathrm{C}$ (above and below the operating temperature). Absence of any degradation in morphology at higher temperatures was apparent in addition to the increase in number density of the pyramidal structures with higher annealing temperatures. The stability of ITO morphology is critical for long-term durability and reliable operation of the sensor at different temperatures. After materials characterization, the sensor response was tested. The response of one of these devices is shown flowing air and the application of $6.5 \mathrm{~V} / 0.77 \mathrm{~A}$ is shown below. The sensors have shown desirable sensitivity and acceptable amounts of signal hysteresis, together with a stable and recoverable baseline.

Figure 4 shows the sensor response study towards the lifetime characterization of the sensor. The absence of any deteriorating response (Figure 3 and 4) of the sensor over time is attributed to a stable three-phase interface (electrode/electrolyte/gas). As the interfacial regions contain the electrochemically active sites, it is vulnerable to micro-structural changes during sensor operation as these regions are also the sites for mass transport, and diffusion. Even well designed interfaces contribute to the electrical losses of devices and hence limit the overall sensor performance. Hence, it is critical to engineer the interface through materials selection and processing for the development of a reliable sensor. 

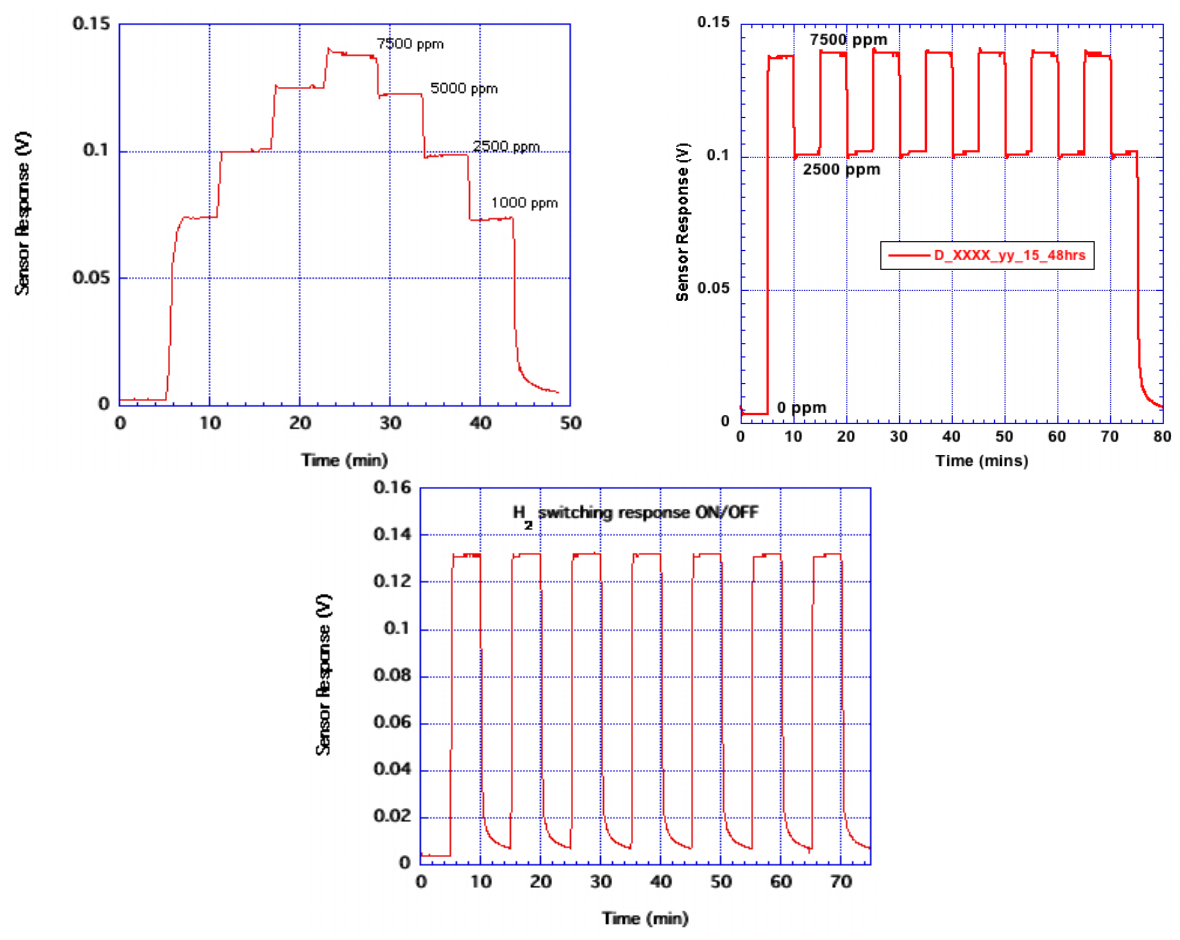

Figure 3. Results from sensor response characterization during life testing. Sensor results were taken self-heated, in flowing air (100-200 $\mathrm{ml} / \mathrm{min})$, using a cold wall containment cell.
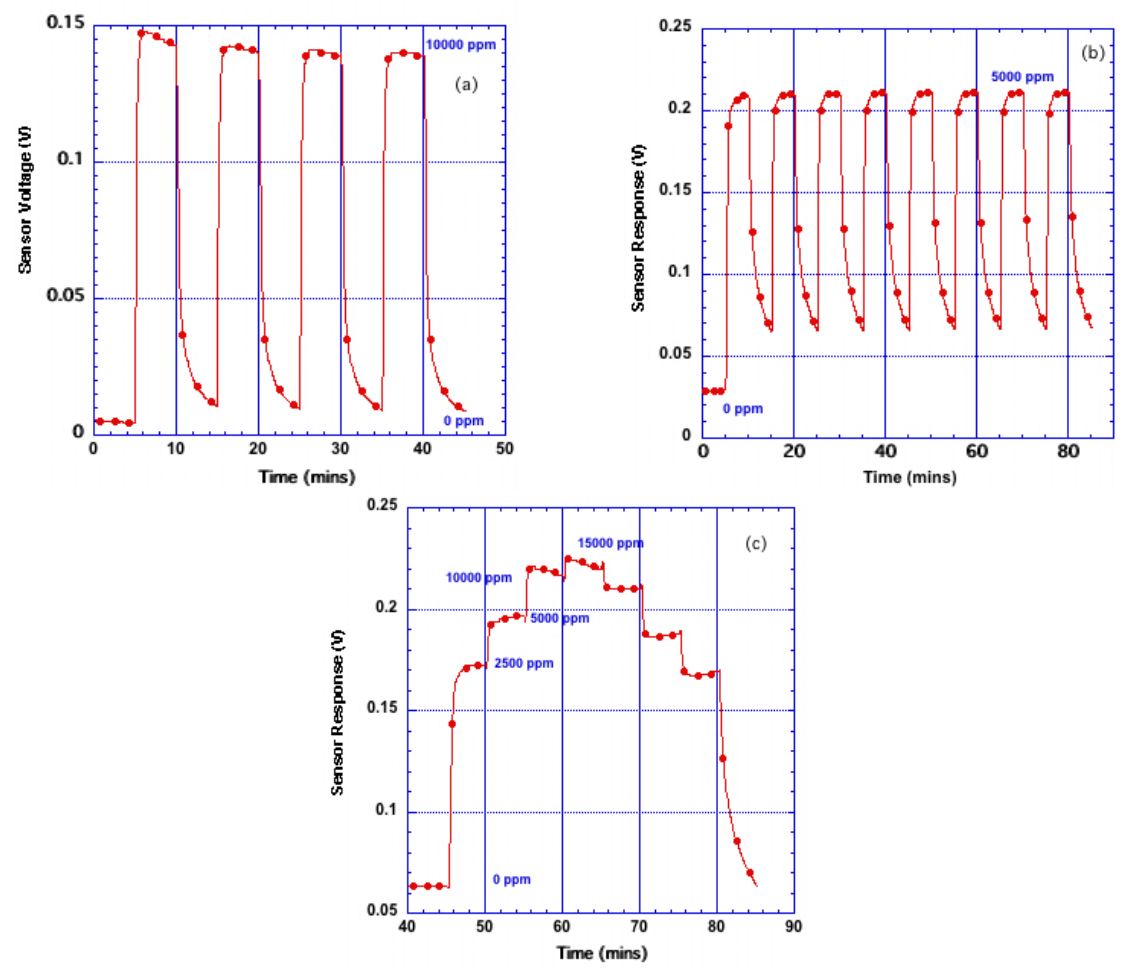

Figure 4. Lifetime testing of the device prototype - sensor response to varying concentrations of $\mathrm{H}_{2}$ at $6.5 \mathrm{~V}$ and $200 \mathrm{SCCM}$ : (a) sensor response to 10,000 ppm of $\mathrm{H}_{2}$ at $492 \mathrm{hrs}$, (b) sensor response to 5,000 ppm of $\mathrm{H}_{2}$ at $795 \mathrm{hrs}$, and (c) sensor response to 2500, 5000, 10000, 15000 ppm of $\mathrm{H}_{2}$ at $949 \mathrm{hrs}$. 
Based on this investigation, the formation of any hydride and its subsequent decomposition needs to be observed, in particular, for high concentration of hydrogen as the presence of hydride induces stress and affects the response rise time. Performing in-situ X-ray diffraction on the sensors, during exposures to various concentrations of hydrogen is envisioned in future. After sensor characterization and optimization, it is imperative to identify challenges toward long-term reliability (over years) and eventually commercialization. The device prototype reported in this article was subjected to over 2000 hrs of testing. Performance testing under rigorous cyclic conditions (temperature ramp and down for 1000 sequences without test gas) is envisioned. Also, sensor ruggedness and durability will be characterized taking onto consideration a relatively packaging scheme and electronic circuitry. Further, a large number of identical sensors will be fabricated and tested to establish statistical confidence.

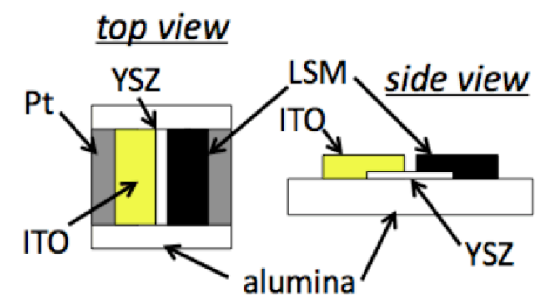

(a)

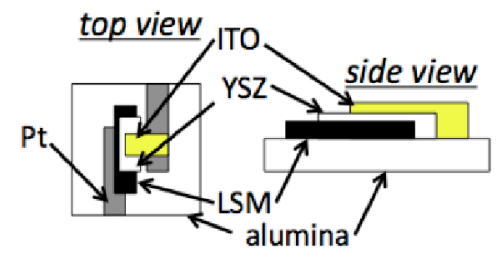

(b)

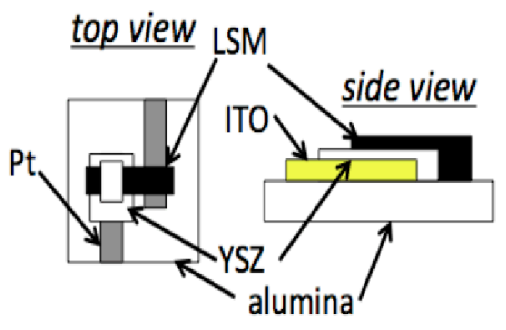

(c)

Figure 5. In-plane (IP) and through-plane (TP) $\mathrm{H}_{2}$ sensor configurations designed by LLNL, (a) IP- dense LSM/YSZ/ITO, (b) TP - dense LSM Bottom/YSZ/ITO top, and (c) TP - ITO bottom/YSZ/dense LSM top.

As a parallel effort, LLNL is investigating different $\mathrm{H}_{2}$ sensor configurations based on strontium-doped lanthanum manganate (LSM) and ITO electrodes. Figure 5 shows the 'inplane' and 'through-plane' sensor configurations where the oxygen ion-conducting path is parallel and perpendicular to the alumina substrate respectively. Upon exposure to varying concentrations of $\mathrm{H}_{2}$, a stable sensor response was observed in the sample using an 'in-plane' configuration with dense LSM. However, the data was noisy attributed to the low ionic conductivity of the porous YSZ. Hence, a transition to a 'through-plane' geometry was desired to increase the ionic conductivity of the porous YSZ and improve the signal-to-noise ratio, while maintaining the better stability that dense LSM. Further testing is in progress to evaluate the aging characteristics of the sensor. 


\section{$\mathrm{H}_{2}$ Level of Storage Sensors for HFCV Applications}

One sensor that the operator of every gasoline or diesel-powered takes for granted is the fuel level gauge or the "gas gauge." The old fashion float and level arm actuating a variable resistor is still being used today among newer technologies based on capacitor plates or a capacitor array. The voltage or current output from these sensors or control boards is used by the vehicle's onboard electronics to provide the operator with instant and almost real-time time information such as fuel remaining and range to empty in some cases.

For conventional compressed-gas hydrogen storage, the pressure of the system can be monitored and if the volume of the system is known, the amount of hydrogen (and therefore vehicle range, range to empty, etc.) may be calculated at any time by an on-board computer. However, the requirement to use ultra-high pressure hydrogen compressed gas cylinders to provide adequate vehicle range has provided significant motivation for interested parties to develop hydride systems that permit safer on-board storage of hydrogen in sufficient quantities to achieve range goals. There is presently no method to determine the amount of hydrogen remaining in a hydride storage system analogous to the liquid level "gas gauge" that gasoline powered vehicles have reliably used for years. Complicating the problem is the fact that, at present, proposed hydrogen storage systems are diverse, and may encompass hydride materials that are in the form of metal solids, liquids, slurries, or may even rely on high surface area sorbants. At present, it is not entirely clear what type(s) of hydride system(s) will be used in future fuel cell vehicles. At present, a principal driver for hydrogen storage system design is storage capacity (and therefore vehicle range) however another important driver is system selection will no doubt come down to be whether sufficient levels of hydrogen purity can be delivered from the storage system given the polymer-based fuel cell's sensitivity to impurities.

While not quite so much as an issue for off-board recharging of hydrides (where the amount of stored hydrogen can be determined by physical weighing a storage cartridge), this diversity of potential hydrogen delivery methods presents a particularly complex problem for those designing vehicle-based systems to measure fuel level for on-board, rechargeable hydride systems. For example, the simplest approach to determine the state of charge of a metal hydride sealed in a steel pressure vessel is to simply weigh the entire cylinder. If the weight of the empty pressure vessel and the weight of the un-hydrogenated metal hydride is known, the exact amount of hydrogen present in the system is known by difference. This simple approach cannot be used when the hydride and pressure vessel are an integral part of a fuel cell vehicle. Furthermore, unlike IC engines, running a fuel cell vehicle to "empty" comes with a significant probability of causing irreversible damage to the fuel cell power plant and therefore will supersede being a mere inconvenience to the FCV operator. A new approach to determine the state of charge of a hydride must be developed. Our initial results in the application of an non-invasive acoustic approach are reported here.

In this approach, we use sound waves to interrogate solid hydride systems. As the amount of hydrogen incorporated into the hydride changes, there will be changes in the multidimensional tensor of elastic constants, or more simply, the stiffness in the hydride material. This change will manifest itself as a change in the speed of sound propagation and also sound speed attenuation since the material is a not perfectly elastic. In this initial work, we wanted to determine if a simple speed of sound or sound speed attenuation measurement using inexpensive and 
commercially available piezoelectric transducers to accurately measure the hydrogen content in the hydride system were possible. In general, ultrasound absorption is a function of frequency in porous materials and more information about the system may easily be obtained by varying the interrogation frequency. Moreover, hydride systems are designed to be porous to maximize rates of hydrogen evolution and to reduce recharge times and porous media are highly nonlinear. Excitation of the hydride system with a single frequency will produce harmonics and complex overtones and this dispersion relation will be a strong function of frequency.

Interestingly, there have been reports in the literature about using sound acoustic methods to in essence "listen" to the acoustic emissions of the pulverization phenomena as the $\mathrm{H}_{2}$ pressure was cycled (8) and it has been acknowledged that the phonon properties and the elastic moduli of many metal hydrides change in response to deformation caused by $\mathrm{H}_{2}$ cycling (9) but there was no indication from the literature that the change in acoustic properties might be used as a gauge of level or state-of-charge. Although commercial ultrasonic-based "level sensors" do exist for powders, these types of devices are contact sensors to determine the level of powder or granular materials in a container and would not work for hydrogen storage. Moreover, there are advantages to developing a non-invasive technology for reasons of safety and cost.

A powerful and well-established technique is swept acoustic frequency interferometery where small changes in physical or chemical properties of a materials system lead to large changes in dispersion and adsorption characteristics of the sound waves. Los Alamos National Lab had pioneered the use of this technique and applied it to non-invasively identify and characterize complex substances such as chemical warfare munitions and nerve agents without direct physical contact with the hazardous substances.

A 300cc 304L stainless steel double-ended cylinder (Swagelock ${ }^{\text {TM }}$ 304L-HDF4-300) was filled with $580 \mathrm{~g}$ of Ergenics 208 metal hydride and conditioned using manufacturer's break-in procedure. A ball valve was attached to the cylinder and the hydride could be charged at 160 psi and subsequently discharged. The mass change between fully charged and discharged conditions was $5.5 \mathrm{~g}$ or just under $1 \%$ of the weight of the hydride. The mass change due to $\mathrm{H}_{2}$ storage was only $0.33 \%$ of the total system weight of the container and hydride taken together. The empty cylinder was laid across 2 piezoelectric transducers along the cylinder axis with a small amount of water-based ultrasonic couplant (SonoTech Ultragel II) in between the vessel and the surface of the transducers. One transducer was driven by a sine wave at $6 \mathrm{~V}$ amplitude and the resonance was recorded with a second piezo transducer separated by approximately 5 inches from the first transducer. Figure 6 shows the response of the hydride cylinder between 100 and $300 \mathrm{kHz}$ in the uncharged state (at 0 psig $\mathrm{H}_{2}$ pressure) and the same experiment repeated after charging the hydride with hydrogen and after a period of time to allow for re-equilibration to room temperature. There is obviously a significant attenuation in the system resonances upon hydrogenation of the metal hydride. The excess hydrogen pressure (charging pressure $=160$ psig) was released to the delivery pressure of the Ergenics 208 (approximately 34 psig) prior to the measurement and before equilibration to ambient temperature.

Although the exact cause of this phenomenon is not known and will require further investigation, it must be related to a change in hydride properties as separate experimentation showed that charging an empty pressure vessel to 34 psig of hydrogen has little effect in the resonance spectra. Furthermore, this attenuation effect is reversible and this suggests that this approach may one day be useful as a level gauge for hydrogen fuel cell storage systems. 

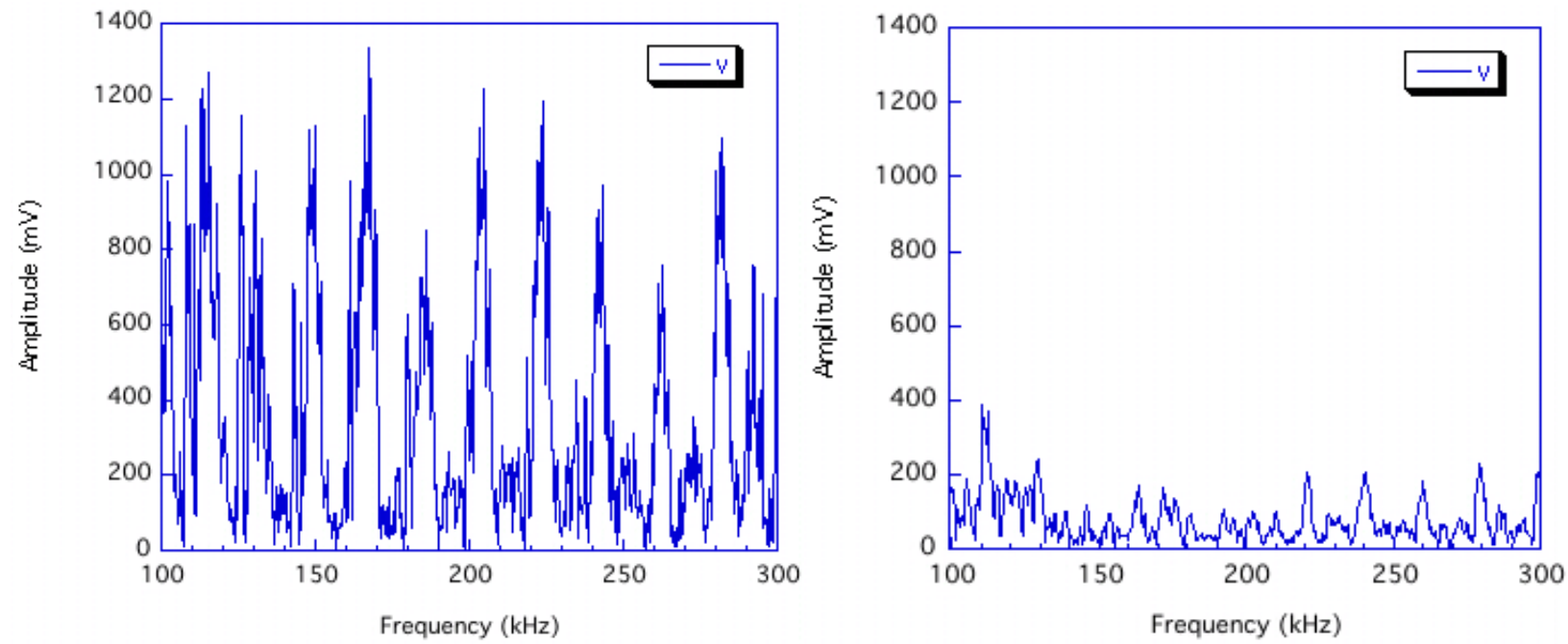

Figure 6. Swept frequency response from 100 to $300 \mathrm{kHz}$ of a 300cc stainless steel pressure vessel containing $580 \mathrm{~g}$ of Ergenics $208\left(\mathrm{MNi}_{4.5} \mathrm{Al}_{0.5}\right)$ hydride powder when uncharged (Left-total vessel and hydride weight $=1643.71 \mathrm{~g})$ and charged (Right) at 160 psi with $5.5 \mathrm{~g}$ of $\mathrm{H}_{2}(1649.20 \mathrm{~g}$ final total weight).

\section{References}

1. L.P. Martin, A-Q. Pham, R.S. Glass, Solid State Ionics 175 (2004) 527.

2. L.P. Martin, A.-Q. Pham, R.S. Glass, J. Electrochem. Soc. 152 (4) (2005) H43.

3. R. Mukundan, E. L. Brosha, and F. H. Garzon, US Patent \# 6,605,202 (2003).

4. R. Mukundan, E. L. Brosha, and F. H. Garzon, US Patent \# 6, 656, 336 (2003).

5. R. Mukundan, E.L. Brosha, F. Garzon, "Mixed Potential Sensors: From Understanding to Applications," ECS Transactions, $208^{\text {th }}$ Meeting of the Electrochemical Society, Los Angeles, CA, (2005).

6. R. Mukundan, E.L. Brosha, and F.H. Garzon, "Tape Cast Sensors and Method for Making," US Patent \# 7,575, 709 (2009).

7. E. Brosha, R. Mukundan, R. Lujan, and F. Garzon, "Development of a Zirconia-Based Electrochemical Sensor for the Detection of Hydrogen in Air," Transactions of the ECS, $214^{\text {th }}$ Meeting, Honolulu, HI, October 2008.

8. S. Didier-Laurant, H. Idrissi, and L. Roue, "In-situ study of the cracking of metal hydride electrodes by acoustic emission technique," J. Power Sources 179 (2008) 412-416.

9. H. K. Birnbaum, "Mechanical Properties of Metal Hydrides," J. Less Comm. Met. 104 (1984)31-41. 


\section{Acknowledgements}

We gratefully acknowledge funding from the US DOE Office of Hydrogen, Fuel Cell and Infrastructure Technologies. We also acknowledge our LLNL collaborators Dr. Robert Glass and Dr. Leta Woo. Portions of this work performed under the auspices of the U.S. Department of Energy by Lawrence Livermore National Laboratory under Contract DE-AC52-07NA27344. Support for hydrogen storage materials/sensor research comes from the Hydrogen Engineering Center of Excellence. 